\title{
Un Análisis Político Comparativo de los Factores de la Estabilidad Política durante la Transición Democrática de Rusia y España a finales del Siglo XX y principios del Siglo XXI
}

\author{
Sergey Boyko, Universidad Estatal de Rusia para las Humanidades, \\ Moscow, Federación de Rusia
}

\begin{abstract}
Resumen: La historia política de Rusia y España ofrece una oportunidad para comparar las medidas adoptadas durante la transición de un régimen autoritario a uno democrático, manteniendo la estabilidad política en el Estado. El Estado es un elemento central del sistema político. La estabilidad del Estado se convierte en una cuestión clave para los dirigentes del Estado, para los sujetos del poder político. La estabilidad política en una democracia requiere la preservación del tipo de organización del poder, que corresponde a los sentimientos e intereses fundamentales socioeconómicos de los grupos sociales. A los factores políticos de la estabilidad pertenece la posibilidad de una participación equitativa en la vida política para todos los estratos sociales, en conformidad con el derecho democrático y las tradiciónes, así como la presencia de las instituciones políticas. El Estado debe ser social y servir al ciudadano y a la sociedad. Se ha demostrado que el Estado debe participar en la regulación de la economía y la circulación monetaria. La estabilidad política en el nivel internacional se logra por el equilibrio multipolar y por la diversidad de los Estados soberanos y equitativos.
\end{abstract}

Palabras Clave: Estabilidad política, Transición democrática, España como ejemplo para Rusia

Abstract: The political history of Russia and Spain offers an opportunity to compare the measures taken during the transition from an authoritarian to a democratic, maintaining political stability in the state. The state is central to the political system. The stability of the state becomes a key issue for the leaders of the State, for the subjects of political power. Political stability in a democracy requires the preservation of the type of organization of power, which corresponds to the fundamental socioeconomic sentiments and interests of social groups. A political factors of stability belongs the possibility of equal participation in political life for all social strata, in accordance with law and democratic traditions, and the presence of political institutions. The State must be socially and serve the citizen and society. It has been shown that the state should participate in the regulation of the economy and currency. Political stability in the international level is achieved by the multipolar balance and diversity of sovereign states and equitable.

Keywords: Political Stability, Democratic Transition, Spain as an example for Russia

$\mathrm{P}$

ARA UN ANÁLISIS comparativo de los problemas del mantenimiento de la estabilidad política dentro de un nivel aceptable en Rusia puede ser útil la experiencia de los países de Europa occidental, donde hay ejemplos en los que se han guardado durante mucho tiempo la estabilidad política y el equilibrio de los intereses de los grupos sociales. Podemos fijarnos en aquellos países en los que se realizó la transición de

Revista Internacional de Ciencias Humanas

Volumen 1, Número 1, www.lascienciashumanas.com, ISSN 2530-4526

(C) Global Knowledge Academics, Sergey Boyko, Todos los Derechos Reservados

Permisos: soporte@gkacademics.com

Republicado de Revista Internacional de Humanidades 1(1), 2012 (pp. 1-8) 
una manera pacífica, contractual, de un régimen político autoritario a un modelo democrático de gobierno estatal.

Las experiencias de América Latina, que a veces son utilizadas como modelo por algunos de los reformadores liberales de Rusia, mostraron su perspectiva débil e incluso suponen una amenaza social para Rusia. Puesto que la estabilidad socioeconómica en estos países no se mantiene, el sistema financiero se encuentra en una condición inestable y la dependencia de la ayuda extranjera supone un problema nacional invencible. Las reformas neoliberales no han producido importantes beneficios económicos, e incluso una estratificación social grave, potencialmente llena de constante inestabilidad política en América Latina, se ha convertido en sistémica. Basta recordar los barrios de las favelas de la ciudad de Río de Janeiro.

Fueron claramente inútiles los intentos de algunos políticos y representantes sociales de Rusia de fines del siglo XX, que trataban de ajustar psicológicamente la realidad de Rusia como estado de poder y potencia periférica; de manera periódica hacían comparaciones entre Rusia y algún país de América Latina, por lo general en los resultados económicos.

Los Estados de la región latinoamericana en su conjunto no tenían un complejo independiente de la industria de guerra, de misiles y armas nucleares, una industria propia de la construcción aeroespacial, una poderosa capacidad científica y tecnológica, una población con el nivel de educación general, especial y superior que cumpliera con los más altos estándares internacionales.

En América Latina nunca ha habido un nivel de bienestar social comparable o mejor que en los Estados de Europa, nunca se ha mantenido la estabilidad política a largo plazo basada en el crecimiento económico sostenido y en el aumento de los niveles y de la calidad de vida. En los sistemas políticos de América Latina no hay ejemplos de avances políticos y económicos complejos, hacia los que Rusia pueda dirigirse en su desarrollo.

En mi opinión, en el contexto del problema analizado, la experiencia más aplicable para Rusia es la experiencia de España. Un país de la Comunidad Europea que sin grandes trastornos alcanzó la posibilidad de efectuar la transición política hacia un Estado democrático y en este camino demostraba el rápido crecimiento socioeconómico y el acuerdo de la gran mayoría de los españoles con el curso de las transformaciones.

Cabe destacar que, a pesar de la actual crisis económica, la estabilidad política en España se mantiene dentro de unos límites democráticamente aceptables.

Asimismo, un fundamento esencial para la pertinencia de las comparaciones de la ciencia política entre Rusia y España puede ser también los múltiples puntos de contacto cultural, histórico, psicológico y emocional entre los pueblos y los Estados de España y Rusia.

Entre Rusia y España nunca ha habido antagonismos ni fuertes enfrentamientos. Durante la Segunda Guerra Mundial, España fue el único país de Europa occidental con un régimen político autoritario que se negó a apoyar oficialmente la agresión militar contra la Unión Soviética. Todo esto en Rusia se recuerda con gratitud.

Analizando lugares comunes en el desarrollo y en el establecimiento de los Estados nacionales y las naciones en estos países, el filósofo y politólogo español José Ortega y Gasset ha señalado acertadamente: "Genial como cultura, fue Grecia inconsistente como cuerpo social y como Estado. Un caso inverso es el que ofrecen Rusia y España, los dos extremos de la gran diagonal europea. Muy diferentes en otra porción de calidades, coinciden Rusia y España en ser las dos razas 'pueblo'; esto es, en padecer una evidente y perdurable escasez de individuos eminentes. La nación eslava es una enorme masa popular sobre la 
cual tiembla una cabeza minúscula. Ha habido siempre, es cierto, una exquisita minoría que actuaba sobre la vida rusa, pero de dimensiones tan exiguas en comparación con la vastedad de la raza, que no ha podido nunca saturar de su influjo organizador el gigantesco plasma popular. De aquí el aspecto protoplasmático, amorfo, persistentemente primitivo que la existencia rusa ofrece. En cuanto a España... Es extraño que de nuestra larga historia no se haya espumado cien veces el rasgo más característico, que es, a la vez, el más evidente y a la mano: la desproporción casi incesante entre el valor de nuestro vulgo y el de nuestras minorías selectas. La personalidad autónoma, que adopta ante la vida una actitud individual y consciente, ha sido rarísima en nuestro país. Aquí lo ha hecho todo el 'pueblo', y lo que el 'pueblo' no ha podido hacer se ha quedado sin hacer. Ahora bien: el 'pueblo' sólo puede ejercer funciones elementales de vida; no puede hacer ciencia, ni arte superior, ni crear una civilización pertrecha de complejas técnicas, ni organizar un Estado de prolongada consistencia, ni destilar de las emociones mágicas una elevada religion"1.

Estas son las evaluaciones unívocas, polémicas, algo ofensivas e injustas hechas por el gran Ortega y Gasset en los años veinte del comienzo del siglo pasado. Sin embargo, proporcionan una oportunidad para comparar los destinos políticos de Rusia y España.

El Estado es un elemento central del sistema político, una institución central del poder. En consecuencia, la estabilidad del Estado se convierte en una cuestión central para los que dirigen el Estado, para los sujetos del poder político. De la estabilidad de los institutos del Estado depende la estabilidad política en el país.

Estabilidad en una democracia requiere la preservación de tal tipo de organización del poder, que corresponde a los sentimientos e intereses fundamentales de los grupos sociales, velando por su interés en el proceso y en los métodos de desarrollo socio-económico. Esto hace que el proceso de desarrollo del país, de la sociedad y del Estado sea eficaz y evolutivo.

Los principales problemas que tuvieron que afrontar los ciudadanos de Rusia tras el colapso de la Unión Soviética y que en el año de 2005 resultaban los más preocupante para los rusos, según encuestas de opinión pública recientes son los siguientes: la pérdida de la estabilidad (57\%), la caída de la moral (56\%), la pérdida del sentido de la seguridad y la confianza en el futuro (50\%), el debilitamiento del orden en el país (44\%), el aumento de los conflictos étnicos $(33 \%)^{2}$.

Todos estos aspectos de la encuesta son, sin duda, son factores -políticos, sociales, económicos, legales- de la determinación de la estabilidad política en el país. Y reflejan las aspiraciones de la mayoría de personas que creen que el poder político está obligado a contar con estas aspiraciones de los ciudadanos para mantener la posición propia estable y confirmar su legitimidad.

Los factores que garantizan la estabilidad política se puede dividir en políticos, administrativos, sociales, económicos, morales, culturales e ideológicos.

A la gama de los factores políticos de la estabilidad pertenece en general la posibilidad de una participación equitativa en la vida política para todos los grupos y estratos sociales, en conformidad con el derecho democrático y las tradiciones, así como la presencia de las instituciones políticas, capaces funcionar efectivamente (no sólo las del Estado y sus instituciones, sino también de la sociedad civil), la disponibilidad y la aplicabilidad de las tecnologías políticas desarrolladas, prácticamente aprobadas.

\footnotetext{
${ }^{1}$ José Ortega y Gasset, España invertebrada, http://juango.es/Ortega y Gasset España invertebrada, pp. 38-39.

${ }^{2}$ El tiempo de las noticias rusas, 11 de marzo de 2005, p. 3.
} 
La autoridad real y legal de la capacidad de cambiar el poder político es uno de los factores determinantes de la estabilidad política; en otras palabras, el factor de la estabilidad política debe incluir un procedimiento legítimo para la transferencia (sucesión) de potencia estatal, incluso en la administración pública.

La historia política de Rusia y España ofrece una gran oportunidad para la comparación y el análisis de las medidas adoptadas durante la transición de un régimen político autoritario a uno democrático, manteniendo al mismo tiempo la estabilidad política en el Estado. La experiencia de España puede ser de interés para Rusia.

En España fue bien pensado y aplicado el mecanismo más o menos aceptable para las principales fuerzas políticas de la transferencia gradual del poder, y fue lograda la meta de la armonía cívica y la estabilidad política.

En la Unión Soviética el proceso de transferencia del poder fue asegurado en el Estatuto del PCUS y en el artículo 6 de la última Constitución soviética. De hecho, todo se basaba en la fuerza del único partido político. No existía el mecanismo de una sucesión política estable, como se refleja en el intento en el año de 1999 del entonces presidente de Rusia Boris Yeltsin para encontrar y preparar a un sucesor suyo. Por supuesto, la Constitución de Rusia y otras leyes definen el procedimiento para elegir al presidente, el parlamento y el gobierno, pero la ley por sí misma no garantiza el camino evolutivo de la transferencia del poder con el consentimiento de las principales fuerzas políticas (partidos, grupos gubernamentales y movimientos).

Marcos institucionales de la estabilidad política son los siguientes:

- la combinación de los poderes del Presidente y de su administración, legislativamente consagrados y reconocidos por los principales grupos sociales, con la autoridad del Gobierno y los poderes del Parlamento (Asamblea Federal);

- un reparto adecuado de las competencias entre el centro federal, los sujetos de la federación y las autoridades locales, formalizado teniendo en cuenta las tradiciones, la experiencia y la racionalidad.

A los factores económicos que contribuyen a la estabilidad política, suelen atribuirse:

- el crecimiento económico sostenido y la transición a la forma social de Estado;

- el crecimiento de los grupos sociales medios (comparables por el nivel de sus ingresos), es decir, el fortalecimiento de la clase media;

- la posibilidad de aplicar los intereses económicos de la mayoría de los grupos sociales bajo la coordinación juiciosa del Estado en el proceso de dicha aplicación.

La práctica ha demostrado que en la cadena "el trabajo-el Estado-las actividades empresariales" el Estado no puede dejar sus posiciones sin poner en peligro la estabilidad política y económica de la sociedad.

Como factor económico de la estabilidad política y señal importante del Estado como una institución política, debe también considerarse la existencia de una propia (nacional) unidad monetaria (divisas) y un derecho existente en exclusiva por parte del Estado de acuñar moneda y de imprimir papel moneda. 
En los estudios de ciencia política este factor de la disponibilidad de una moneda propia como señal de estabilidad del Estado no está aún debidamente argumentado y justificado. Tal vez porque esta cuestión tiene un carácter científico interdisciplinario.

El sociólogo y politólogo estadounidense Seymour Lipset, uno de los fundadores de la teoría de la modernización, ha investigado exhaustivamente la dependencia de la estabilidad política respecto de la legitimidad del poder estatal, y como ejemplo establece una analogía entre la legitimidad de la autoridad política y la credibilidad de la unidad monetaria nacional.

Esta conclusión de S. Lipset puede ser interpretada como un ejemplo de la dependencia de la estabilidad política respecto de la estabilidad de la circulación monetaria, en tanto que justificación de un punto nodal en la estabilidad del Estado ${ }^{3}$.

En la historia del desarrollo y del establecimiento de todos los Estados podemos hallar ejemplos de la formación, desarrollo y regulación estricta de la circulación monetaria nacional como un elemento importante del Estado y de la sociedad.

El Estado es propietario del derecho de regular la circulación monetaria en el país. La estabilidad del sistema monetario es un elemento clave de la estabilidad política.

El control fiscal y monetario sólo es posible por parte de las autoridades públicas. La estabilización de la unidad monetaria, la planificación y la reducción de la inflación son algunos de los principales objetivos de la política macroeconómica.

Las forma de la propiedad en el país, las políticas económicas y la regulación estatal de la economía pueden ser diferentes.

Sin embargo, la regulación de la circulación monetaria es prerrogativa exclusiva del Estado. En el caso de la moneda del euro son los organismos pertinentes de la Unión Europea. La estabilidad política depende directamente de la estabilidad de la circulación monetaria.

Los factores sociales necesarios son: un nivel aceptable de cultura política, lo que permite una forma civilizada de resolver los conflictos que surgen de los intereses contrapuestos; la tolerancia; las tendencias democráticas; el respeto de la ley; el cumplimiento de las normas acordadas en el proceso político; el desarrollo de las instituciones políticas reconocidas por la ley.

El principal factor social es seguir el principio de que "el Estado es para el ciudadano, para la sociedad y el pueblo". La Constitución de Rusia (artículo 7, párrafo 1) establece: "La Federación de Rusia es un estado social cuya política está dirigida a crear las condiciones para asegurar una vida digna y un desenvolvimiento libre del ciudadano"4.

En las primeras palabras de la Constitución de España (título preliminar, artículo 1) se determina que: "España se constituye en un Estado social y democrático de Derecho, que propugna como valores superiores de su ordenamiento jurídico la libertad, la justicia, la igualdad y el pluralismo político",5.

Los factores legales de la estabilidad son los principios, normas y reglamentos de la división democrática de los poderes del gobierno estatal. Las leyes electorales y los procedimientos para la transferencia de poder en el país, controlados por las autoridades estatales, también son observados y protegidos estrictamente por las fuerzas de seguridad, así como están reconocidos por la sociedad civil; es decir, tienen una legitimidad política en el país.

\footnotetext{
${ }^{3}$ S. Lipset, Consensus and Conflict: Essays in Political Sociology, New Brunswick, New Jercey, 1985, p. 23.

${ }^{4}$ Constitución de la Federación de Rusia, edición oficial, Moscú, 2010, p. 6.

${ }^{5}$ Spanish Constitution and standing orders of the Congress, Madrid, 1990, p. 10.
} 
En el caso de Rusia, en el campo de la ideología, un factor de estabilidad política puede ser una transición a las ideas del conservadurismo, o más bien, del tradicionalismo. En la historia política de Rusia el conservadurismo (tradicionalismo) jugó un papel progresista. Siempre que se implementaban los programas del desarrollo gradual conservador progresista, Rusia lograba grandes éxitos en el ámbito socioeconómico. Se fortalecía su situación interna, el país se consolidaba, aumentaba su influencia política y el prestigio internacional.

No se puede vivir en el pasado, pero las experiencias nacionales positivas deben ser consideradas.

Se ha demostrado repetidamente que la copia directa de la experiencia extranjera conduce a un resultado contrario al esperado. Puede tener lugar una fuerte desaceleración en el desarrollo económico y social, así como la caída de la calidad de vida de la población, la desestabilización del sistema político y el descrédito de los principios democráticos de gobierno del Estado.

El conservadurismo puede ser democrático, así como el liberalismo puede ser reaccionario para un país.

Entre los factores de la administración ejecutiva que aseguran la estabilidad política es posible destacar los siguientes:

- la disponibilidad del orden constitucional apoyado por las autoridades y la legitimidad del actual régimen político y administrativo;

- la flexibilidad en el uso legítimo de los medios coercitivos del poder, el apoyo por los grupos sociales de base (por la mayoría de los ciudadanos) de los órganos de aplicación de la ley de la autoridad estatal;

- el cumplimiento de las tradiciones sociales y las prioridades culturales y religiosas;

- la ausencia de amenaza de grandes cambios estructurales en la organización del poder del Estado, capaces conducir al cambio de régimen político;

- la disponibilidad y la aplicación coherente de las estrategias gubernamentales públicas saneadas y eficaces;

- el mantenimiento sostenible de las relaciones democráticas tolerantes entre las autoridades públicas y la oposición;

- la realización práctica por el gobierno nacional de las funciones básicas constitucionales y legislativamente fijadas de manera eficaz y eficiente.

El resultado de mantener la estabilidad política se expresa en los resultados de la votación en elecciones democráticas periódicas, ya sea con el apoyo o con el cambio de un partido político que ejerce las funciones de poder estatal público y la gobernabilidad en el país.

Los factores globales de la estabilidad política se manifiestan en el siguiente gobierno. Como demuestra la experiencia histórica de la humanidad, el mundo unipolar en el que un Estado o un pequeño grupo de Estados dominan políticamente, a largo plazo y con estabilidad, no funciona. E incluso puede terminar mal en el caso de que se pase a la argumentación armada de su punto de vista en las relaciones internacionales.

La estabilidad política se logra por el equilibrio multipolar de los poderes y por la diversidad de los Estados soberanos y equitativos. Entre los ejemplos recientes se puede destacar la creación de un grupo internacional de los Estados BRICS: Brasil, Rusia, India, China, República Sudafricana. 
Es difícil poder combinar en una sola organización internacional a los Estados que más se diferencian por su pasado histórico, por la composición étnica y por la cultura nacional, así como por los caminos sociales y económicos atravesados.

Los Estados BRICS se han unido con el deseo de crear una base para el potencial económico común (que es realmente impresionante), un centro de desarrollo independiente y soberano en beneficio de sus propios pueblos. Los Estados BRICS no crean obstáculos para el bienestar de otros Estados y el mundo.

\section{Sobre el Autor}

Dr. Sergey Boyko

Nació en 1952. Se graduó en la facultad de la economía y derecho de la Universidad de la amistad de los pueblos en Moscú. Economista. Postgraduado en la Academia de Administración Pública de la Presidencia de la Federación de Rusia y en la Academia de relaciones sociales. Politólogo, doctor en ciencias políticas. Trabajaba en las organizaciones del comercio exterior, en los ministerios, en las organizaciones no gubernamentales. Los últimos 10 años trabaja como funcionario público (referente jefe) en el Aparato de la Duma Estatal (Parlamento) de la Federación de Rusia. En paralelo se dedica de la enseñanza de ciencias políticas en la Universidad Estatal de Rusia para las Humanidades. Líneas de interes y investigación científica: la estabilidad política en el Estado, la evolución de los partidos políticos, el tránsito político hacia la democracia, los problemas del Estado contemporáneo y la administración pública. 